\title{
Analytical solution for katabatic flow induced by an isolated cold strip
}

\author{
Simon L. Axelsen • Alan Shapiro • Evgeni Fedorovich • \\ Han van Dop
}

Received: 8 April 2009 / Accepted: 13 November 2009 / Published online: 25 November 2009

(C) The Author(s) 2009. This article is published with open access at Springerlink.com

\begin{abstract}
An analytical model for katabatic flow induced by cold strip of finite width in the cross-slope direction but of infinite extent in the downslope direction is presented. The fluid is assumed to have a constant (eddy) viscosity, and the Coriolis force is neglected. A numerical simulation has been used to verify the model, which is shown to revert to the classical Prandtl model if the strip width goes to infinity. The effects of the strip width and slope angle on the katabatic flow are studied. The buoyancy and downslope velocity reach maximum values at the centre of the strip, and spread outwards in the cross-slope direction. The downslope wind maximum weakens for narrow strips and shallow slopes. In contrast to the Prandtl solution, which shows a counter flow above the wind maximum, our model predicts the counter flow to occur outside the strip. The cross-slope variation in the surface forcing induces cross-slope and slope-normal velocities, which are manifested in vortices at the strip edges. Below the wind maximum, the fluid above the cooling surface descends and moves toward the strip edge where it is detrained from the strip region. Replenishment of fluid into the strip region takes place above the wind maximum.
\end{abstract}

Keywords Katabatic flow $\cdot$ Inhomogeneous surface forcing $\cdot$ Analytical solution

\section{Introduction}

It is well known that the local weather in mountainous regions is (partly) determined by the thermally driven slope winds. These downslope (katabatic) and up-slope (anabatic) winds arise when there is a horizontal temperature difference between the air at the surface and the environmental air at the same altitude. Solar heating during the day and radiative cooling

S. L. Axelsen $(\varangle) \cdot$ H. van Dop

Institute for Marine and Atmospheric Research Utrecht (IMAU), Utrecht University, P.O. Box 80.005,

3508 TA Utrecht, The Netherlands

e-mail: s.1.axelsen@uu.nl

A. Shapiro $\cdot$ E. Fedorovich

School of Meteorology, University of Oklahoma, Norman, OK, USA 
during the night contribute to this temperature difference. In the case of a negative (positive) temperature difference, the associated negative (positive) buoyancy force has a component in the downslope (up-slope) direction and induces a katabatic (anabatic) flow.

In this article we focus on katabatic winds over glaciers, often called glacier winds. They are characterized by wind profiles exhibiting persisting wind maxima of the order of several meters per second. The wind maximum is normally located a couple of metres to tens of metres above the surface. Turbulence in the katabatic layer is produced by wind shear. It is responsible for the vertical mixing of momentum and heat in the lowest part of the boundary layer, and effectively transports heat from the boundary layer to the surface. In the ablation zone of glaciers, warming of the surface causes melting. On a regional scale, glaciers supply melt water for hydropower reservoirs and for irrigation systems [1].

Slope flows are subject to inhomogeneous forcings of various types. Inhomogeneous surface buoyancy fields may be due to partial cloud cover, differential solar heating, difference in surface cover (e.g. snow/soil/water) and variations in the vegetation types. Katabatic flows over the ablation zones of glaciers are affected by a downslope variation in the surface buoyancy. While the temperature of the ice is constant at $0^{\circ} \mathrm{C}$, the corresponding potential temperature decreases down the slope, typically at a rate exceeding the rate of change of the environmental potential temperature.

In addition to inhomogeneous surface buoyancy fields, katabatic winds in mountainous terrain are affected by the ubiquitously changing orography. The downslope flow over a valley glacier may be, for instance, influenced by a narrowing/widening of the valley and changes in the valley direction. The valley walls form (ice-free) impenetrable boundaries for the glacier wind. When they are heated, the temperature difference between the walls and the centre of the valley generates a cross-slope circulation [2]. The characteristics of katabatic flows are further influenced by a variety of factors such as ambient winds, the slope angle, and the stability of the environmental air.

The katabatic flow over a sufficiently long slope is approximately one-dimensional, i.e. the flow depth, speed and buoyancy are invariant with downslope distance [3-5]. This invariance simplifies the governing equations, permitting the vertical structure to be analytically described. The classical Prandtl model [6] considers the katabatic flow along a uniformly cooled sloping planar surface in a stably stratified fluid. In the downslope momentum equation, the downslope component of buoyancy is opposed by friction, and in the buoyancy equation the cooling induced by the surface is compensated by the along-slope advection of warm air. The resulting vertical profile of buoyancy shows a layer of negative buoyancy near the surface, capped by a layer of positively buoyant air (further aloft the oscillation in the profile around zero buoyancy quickly dampens out). The associated profile of downslope velocity exhibits a low-level wind maximum, topped by a weak reverse flow. Observations of katabatic flow show that an appropriately tuned Prandtl model qualitatively reproduces the observed profiles of downslope velocity and buoyancy rather well $[5,7]$.

Several extensions to the one-dimensional Prandtl model have been proposed. In $[7,8]$, height-dependent turbulence exchange coefficients were introduced, yielding an analytical solution valid in the WKB approximation. The model was further extended in [9] to include time dependence. Other related models have made provision for the Coriolis force (e.g. [1015]), some in combination with external winds, radiative damping and time dependence. The various models treat the exchange coefficients differently, from constants to more complex height dependent functions. As shown in [10], the buoyancy and cross-slope velocity fields tend to spread inexorably upward when the Coriolis force is included. It was argued in [13] that these fields vanish away from the slope when either a geostrophic wind or a radiative damping is taken into account. However, in the case of an imposed geostrophic wind, 
unrealistically large values of those winds were required to prevent the continued upward growth of the buoyancy and cross-slope velocity fields. More recently, it was argued in [15] that the upward growth would diminish if the turbulence exchange coefficients are chosen appropriately, a result questioned in the review by [16].

The effect of topography on slope flow was studied by [17], who looked at the linkage of the flow in a main valley and the circulation in sidevalleys. Slope winds induced by differential surface buoyancy fields have been studied in e.g. [18-20]. Authors of [16] (hereafter SF08) looked at the katabatic flow induced by an along-slope cold strip of limited width. The flow problem additionally included the Coriolis force. The qualitative behaviour of the cross-slope velocity and buoyancy fields far above the surface was investigated analytically. However, closer to the surface the buoyancy and velocity fields were inferred from numerical simulations. Numerical results showed two counter-rotating circulations on either side of the strip, centred on baroclinic zones of strong cross-slope surface buoyancy gradient. A broad region of descending fluid was found between the two circulation centres. Throughout the lower part of the domain, a pronounced cross-slope flow was observed.

In contrast to one-dimensional studies, SF08 found that for katabatic winds induced by an isolated strip, the cross-slope velocity and the buoyancy did not diffuse upward indefinitely. They also found that the downslope and slope-normal velocities would not vanish far above the surface. Instead, the far-above-slope downslope and slope-normal velocities combined into a purely horizontal streaming motion along environmental isentropes. It was argued in SF08 that secondary processes not included in the equations may, if persistent, be vital in controlling the structure of the steady-state flow.

To our knowledge, no analytical solution has yet been obtained for the katabatic flow induced by a surface buoyancy forcing that is inhomogeneous in the cross-slope direction. In this paper we extend the Prandtl model to include such an inhomogeneous surface forcing. The work builds on the considerations by SF08, except that we consider glaciers winds (characterized by short time scales and shallow boundary layer depths) for which the Coriolis force plays a negligible role.

The flow problem and solution are formulated in the next section. In Sect. 3 we verify the analytical model by comparing model results to results obtained from numerical simulation. The effects of the strip width and slope angle on the spatial structure of the buoyancy and velocity fields are discussed in Sect. 4 . We limit the consideration to slope angles typical of glaciers (e.g. $\left.1-10^{\circ}\right)$. Finally, in Sect. 6 we present a summary and suggestions for future work.

\section{Analytical model}

The katabatic flow is described in a Cartesian coordinate system aligned with the slope, which is inclined at an angle $\alpha(>0)$ to the horizontal. The $x, y$ and $z$ axes point respectively along the flow direction, the cross-slope direction, and the slope-normal direction, see Fig. 1.

In the rotated coordinate system, the Boussinesq equation of thermodynamic energy is

$$
\frac{\partial b}{\partial t}+(\mathbf{v} \cdot \nabla) b=N^{2}(u \sin \alpha-w \cos \alpha)+\kappa \nabla^{2} b .
$$

Here $b$ is the buoyancy, the Laplace operator is $\nabla^{2}=\partial^{2} / \partial x^{2}+\partial^{2} / \partial y^{2}+\partial^{2} / \partial z^{2}, \kappa$ is the thermal diffusivity coefficient, $N=\left(g \Gamma / \theta_{r}\right)^{1 / 2}$ is the Brunt-Väisälä frequency with $g$ and $\theta_{r}$ being, respectively, the acceleration due to gravity and a reference potential temperature, and $\Gamma \equiv \mathrm{d} \Theta_{a} / \mathrm{d} z^{*}$ is the constant vertical gradient of the ambient potential temperature, $\Theta_{a}$, 


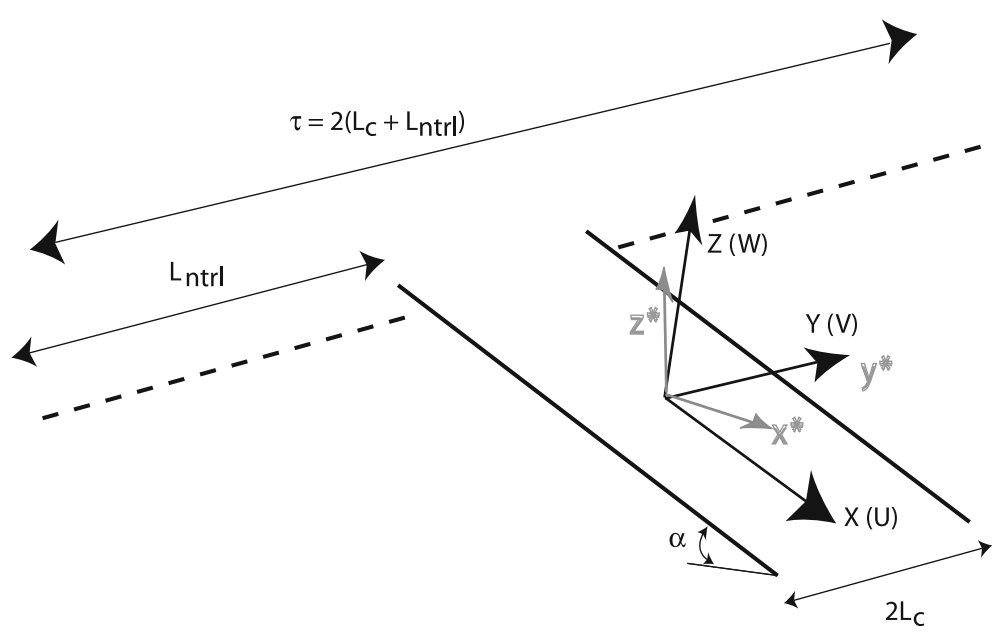

Fig. 1 The slope-following Cartesian coordinate system is rotated by the slope angle $\alpha(>0)$ to the horizontal, non-rotated Cartesian coordinate system denoted by asterisk. The downslope flow, induced by an isolated cold strip with width $2 L_{c}$, is in the $X$-direction. The surface buoyancy field is inhomogeneous in the cross-slope $(Y)$ direction: adjacent to the strip is a neutrally buoyant surface with width $L_{n t r l}\left(L_{n t r l} \rightarrow \infty\right.$ for an isolated strip). All quantities are dimensionless, see text for further details

in the non-rotated coordinate system, denoted by an asterisk. The velocity vector $\mathbf{v}$ has the components $u, v$ and $w$ in the $x, y$ and $z$ directions, respectively. The momentum balance and continuity equations are

$$
\begin{aligned}
& \frac{\partial u}{\partial t}+(\mathbf{v} \cdot \nabla) u=\frac{-1}{\rho_{r}} \frac{\partial p^{\prime}}{\partial x}-b \sin \alpha+v \nabla^{2} u, \\
& \frac{\partial v}{\partial t}+(\mathbf{v} \cdot \nabla) v=\frac{-1}{\rho_{r}} \frac{\partial p^{\prime}}{\partial y}+v \nabla^{2} v, \\
& \frac{\partial w}{\partial t}+(\mathbf{v} \cdot \nabla) w=\frac{-1}{\rho_{r}} \frac{\partial p^{\prime}}{\partial z}+b \cos \alpha+v \nabla^{2} w, \\
& \nabla \cdot \mathbf{v}=0,
\end{aligned}
$$

where $v$ is the diffusion coefficient of momentum, and $\rho_{r}$ is a constant reference density. The perturbation pressure $p^{\prime}=p-p_{a}$ is the pressure $p$ minus the hydrostatic pressure $p_{a}$, which is dependent only on $z^{*}$.

In the remainder we use the non-dimensionalized variables introduced in SF08, repeated here for convenience:

$$
(X, Y, Z) \equiv \frac{(x, y, z)}{l_{s}}, \quad(U, V, W) \equiv \frac{(u, v, w)}{u_{s}}, \quad \Pi \equiv \frac{p^{\prime}}{p_{s}}, \quad B=\frac{b}{b_{s}},
$$

where the length, pressure, velocity and buoyancy scales are given, respectively, by

$$
l_{s} \equiv\left(\frac{v}{N \sin \alpha}\right)^{1 / 2}, \quad p_{s} \equiv \rho_{r} l_{s} b_{s} \cos \alpha, \quad u_{s} \equiv \frac{b_{s}}{N}, \quad b_{s} \equiv\left|b_{0}\right|=|b(z=0)| .
$$

Following SF08, we consider a stationary flow that is homogeneous in the downslope direction (all derivatives with respect to $X$ drop out, e.g. the Laplacian becomes $\nabla^{2}=\partial^{2} / \partial Y^{2}+$ $\left.\partial^{2} / \partial Z^{2}\right)$. Linearizing (1)-(5) yields 


$$
\begin{aligned}
& 0=U-W \cot \alpha+P^{-1} \nabla^{2} B, \\
& 0=-B+\nabla^{2} U, \\
& 0=-\frac{\partial \Pi}{\partial Y} \cot \alpha+\nabla^{2} V, \\
& 0=-\frac{\partial \Pi}{\partial Z} \cot \alpha+B \cot \alpha+\nabla^{2} W, \\
& 0=\frac{\partial V}{\partial Y}+\frac{\partial W}{\partial Z},
\end{aligned}
$$

where $\operatorname{Pr} \equiv v / \kappa$ is the Prandtl number. This set of equations is similar to Eqs. 5.1-5.5 is SF08. One difference is the absence of the Coriolis term, which links the downslope velocity to the cross-slope velocity, and vise versa. Secondly, we do not assume the length scales in the cross-slope direction to be much larger than the length scales in the vertical direction (i.e. $\left.\partial^{2} / \partial Y^{2} \ll \partial^{2} / \partial Z^{2}\right)$.

Above we used $l_{s}$ as a scale factor for both the slope-normal and cross-slope distances.

This was done for the sake of convenience, and the reader should bear in mind that we do not intend to imply that any particular terms are dominant or that other terms are negligible. The only approximations made in the analysis are the Boussinesq approximation and the neglect of the nonlinear perturbation terms.

By linearizing the governing equations, the advection terms dropped out. Below we show that for laminar flows, at very low Reynolds numbers, this approximation is justifiable. For turbulent flows, however, the approach is more questionable. This issue is addressed in Sect. 6.

Equation 10 permits the introduction of a stream function $\Psi$ defined as $V=\partial \Psi / \partial Z$ and $W=-\partial \Psi / \partial Y$. The pressure is eliminated by taking the $Z$-derivative of (8) and subtracting the $Y$-derivative of (9), to obtain

$$
\begin{aligned}
& 0=U+\frac{\partial \Psi}{\partial Y} \cot \alpha+\nabla^{2} B \\
& 0=-B+\nabla^{2} U \\
& 0=-\cot \alpha \frac{\partial B}{\partial Y}+\nabla^{4} \Psi .
\end{aligned}
$$

Here we assume for convenience that $\operatorname{Pr}=1$, thus we assume that $\nu$ and $\kappa$ are constant ${ }^{1}$ exchange coefficients whose ratio is unity. We do not expect that changes to the Prandtl number will greatly influence the results: in [22], laminar slope flows of a stably-stratified fluid along a vertical plate were investigated, and it was found that the results did not change much in going from $P r=1$ to $P r=1.5$. Also, the classical Prandtl solution has a $1 / 4$ power dependence on $\mathrm{Pr}$, which is rather weak for $\mathrm{Pr} \sim 1$. These arguments make it reasonable to use $\operatorname{Pr}=1$ in this study.

The system of Eqs. 11-13 is further reduced to

$$
\left[\nabla^{6}+\nabla^{2}+c \frac{\partial^{2}}{\partial Y^{2}}\right] B=0,
$$

where $c \equiv \cot ^{2} \alpha$.

We solve Eqs. 11-13 by using Fourier series and the fact that the buoyancy, downslope velocity and stream function are assumed to be periodic in a horizontal domain with width

$$
\tau=2 L_{c}\left(1+L_{n t r l} / L_{c}\right)
$$

1 This assumption is a simplification of reality. Observations of turbulent flows have shown that the Prandtl number depends on stability, and that $\operatorname{Pr}>1$ in the SBL, see e.g. [21] and references herein. 
See Fig. 1, the position of the domain within the coordinate system is chosen in a way that $Y=0$ is in the middle of the strip. The non-dimensional half-width of the negatively buoyant surface, $L_{c}$, is obtained by scaling the dimensional half-width of the strip by $l_{s}$. Note, although $L_{c}$ is the half-width of the strip, we shall in the remainder refer to this variables as the strip width. The non-dimensional width of the neutrally buoyant surface, $L_{n t r l}$, is defined in a similar way.

Since we use Fourier series to solve the flow problem analytically, we must apply periodic boundary conditions in the cross-slope direction. This periodicity implies that there is an infinite number of isolated strips. However, if the neutrally buoyant surface separating a given strip from its neighbours is very broad, then the neighbouring strips should only minimally influence the flow over the given strip.

For boundary conditions, we require the buoyancy and velocity fields to vanish far above the surface, i.e. $B, U, \Psi \rightarrow 0$ as $Z \rightarrow \infty$. At the surface we impose the no-slip condition to the downslope and cross-slope velocities, the impermeability condition to the slope-normal velocity, and prescribe the buoyancy, which depends on the cross-slope location. The nondimensional surface buoyancy in the strip region is $B\left(\left|Y / L_{c}\right| \leq 1, Z=0\right)=-1$, and for the neutrally buoyant surface $B\left(\left|Y / L_{c}\right|>1, Z=0\right)=0$.

It can be seen that this boundary value problem (differential equations and boundary conditions) has only two degrees of freedom: the slope angle $\alpha$, and the non-dimensional length scale characterizing the width of the cold strip $L_{c}$. There is a third parameter, the ratio of the strip width to the width of the computational domain, but our intention is to consider strips that are isolated (as much as possible) so this ratio will eventually drop from the problem. In practice, it will be made as small as computational resources will allow. Note that none of the non-dimensional governing parameters of the problem can be interpreted as a Reynolds number, which should be expected since the nonlinear terms have been omitted.

We solve (14) by expanding $B$ as

$$
B(Y, Z)=\sum_{n=1}^{\infty}\left[a_{n}(Z) \cos \left(\frac{2 n \pi}{\tau} Y\right)+\tilde{a}_{n}(Z) \sin \left(\frac{2 n \pi}{\tau} Y\right)\right]+a_{0}(Z) .
$$

We introduce the new variable $p \equiv n \pi /\left[L_{c}(1+\mathcal{R})\right]$, where the isolation parameter is defined as

$$
\mathcal{R} \equiv L_{n t r l} / L_{c}
$$

It varies from 0 (Prandtl model, cooling over the entire slope) to $\infty$ (an isolated cold strip). Due to the choice of coordinate system and surface boundary conditions, $B$ is an even function in $Y$, which gives $\tilde{a}_{n}=0 \forall n$. Below we sketch how the general solution to (11)-(13) is obtained, with the detailed derivation being given in Appendix A.

Since (14) is a linear homogeneous differential equation with constant coefficients, we anticipate that it admits exponential solutions. Accordingly, in (16) we assume that $a_{n}(Z) \propto$ $e^{m Z}$, yielding a sixth-order ordinary differential equation in $Z$. The proportionality constants associated with $a_{n}$ are determined by the boundary conditions. The general solution to (14) is (see also Eq. A.3)

$$
B(Y, Z)=\sum_{n=1}^{\infty} \cos (p Y)\left[C_{n} e^{m_{1} Z}+D_{n} e^{m_{2} Z}+E_{n} e^{m_{3} Z}\right]+a_{0}(Z),
$$

with $C_{n}, D_{n}$ and $E_{n}$ being constants determined by the boundary conditions. By the requirement that the buoyancy vanishes far above the surface, only three exponents $m_{i}$ are retained, 
see Eq. A.2. The $n=0$ contribution to the Fourier series is discussed separately in Appen$\operatorname{dix} \mathrm{C}$.

Regarding $B$ as known, the stream function $\Psi$ can be determined from (13), which is a fourth-order, linear, non-homogeneous differential equation. The solution is the sum of a particular solution (see Eq. A.5) and the solution to the homogeneous part (see Eq. A.7):

$$
\Psi(Y, Z)=\sum_{n=1}^{\infty} \sin (p Y)\left[\beta_{1, n} C_{n} e^{m_{1} Z}+\beta_{2, n} D_{n} e^{m_{2} Z}+\beta_{3, n} E_{n} e^{m_{3} Z}+\gamma_{n} e^{-p Z}\right] .
$$

Because the expansion is carried out over sines, the $n=0$ contribution to the Fourier series for $\Psi$ is zero. The constants $\beta_{\sigma, n}$ in Eq. A.6, are associated with the particular solution and relate the stream function to the buoyancy. The constants $\gamma_{n}$ are associated with the solution to the homogeneous differential equation and are determined by the boundary conditions.

Lastly, the downslope velocity $U$ is found using Eq. 12. The general solution is obtained using the same procedure as for the stream function, i.e. finding a particular solution (see Eq. A.9) and adding the solution to the homogeneous differential equation (see Eq. A.11):

$$
\begin{aligned}
U(Y, Z) & =\sum_{n=1}^{\infty} \cos (p Y)\left[\delta_{1, n} C_{n} e^{m_{1} Z}+\delta_{2, n} D_{n} e^{m_{2} Z}+\delta_{3, n} E_{n} e^{m_{3} Z}+\epsilon_{n} e^{-p Z}\right] \\
& +(n=0 \text { contribution }) .
\end{aligned}
$$

The constants $\delta_{\sigma, n}$ relate the downslope velocity to the buoyancy, see Eq. A.10, whereas $\epsilon_{n}$ are constants associated with the solution to the homogeneous differential equation and determined by the surface boundary condition.

In total five constants are determined by the surface boundary conditions. The surface buoyancy $B_{s}$, which is -1 in the strip region and 0 outside, is expanded in a Fourier series with Fourier coefficients $\mathcal{B}_{n}$, see Eq. B.1. The no-slip condition is applied to $U$ and $V$ (= $\partial \Psi / \partial Z$ ), while the impermeability condition is applied to $W(=-\partial \Psi / \partial Y)$. Lastly, we use the Fourier representation of $U, \Psi$ and $B$ in (11). The resulting equations, which are worked out in Appendix B, can be summarized in a matrix form as

$$
\left(\begin{array}{ccccc}
1 & 1 & 1 & 0 & 0 \\
\delta_{1} & \delta_{2} & \delta_{3} & 1 & 0 \\
m_{1} \beta_{1} & m_{2} \beta_{2} & m_{3} \beta_{3} & 0 & -p \\
\beta_{1} & \beta_{2} & \beta_{3} & 0 & 1 \\
v_{1} & v_{2} & v_{2} & 1 & p \sqrt{c}
\end{array}\right)\left(\begin{array}{c}
C_{n} \\
D_{n} \\
E_{n} \\
\epsilon_{n} \\
\gamma_{n}
\end{array}\right)=\left(\begin{array}{c}
\mathcal{B}_{n} \\
0 \\
0 \\
0 \\
0
\end{array}\right),
$$

where $v_{\sigma}=\delta_{\sigma, n}+p \beta_{\sigma, n} \sqrt{c}+m_{\sigma}^{2}-p^{2}$ (no summation over $\sigma$ ).

For $n=0$ the constants $\delta_{\sigma, n}$ and $\beta_{\sigma, n}$ become singular. In Appendix $\mathrm{C}$ we look at the $n=0$ contribution to the Fourier series (18) and (20), and find that these first terms yield solutions to the buoyancy and downslope velocity fields that are proportional to the Prandtl solution. In Sect. 4, the strip-centre vertical profiles of buoyancy and downslope velocity are compared to the Prandtl solution, which in a non-dimensional form reads:

$$
\begin{aligned}
& B_{P r}(Z)=-e^{-Z / \sqrt{2}} \cos (Z / \sqrt{2}), \quad \text { and } \\
& U_{P r}(Z)=e^{-Z / \sqrt{2}} \sin (Z / \sqrt{2}) .
\end{aligned}
$$

In Appendix $\mathrm{C}$ we show that for the homogeneous slope $(\mathcal{R}=0)$, the new analytical solution reverts to the Prandtl solution. 


\section{Model verification}

Although our analytical model reverts to the Prandtl model in the case $\mathcal{R}=0$, it is desirable to verify the model for a non-zero $\mathcal{R}$. In this section we present a comparison of the analytical model with results from a direct numerical simulation (DNS) of a laminar katabatic flow.

A general introduction to DNS can be found in e.g. [23]. Our numerical code is based on the works [22,24-26]. The adaptation of the code to simulation of flow over an isolated strip is described in SF08. The reader is referred to these articles for a further description of the model; here we only give a short summary.

The governing Eqs. 1-4 are solved on a staggered Cartesian grid with a uniform spacing in a rectangular domain. The spatial derivatives in the advection and diffusion terms are discretized with a second-order finite difference expression. The time integration of these terms, as well as the buoyancy term, is calculated using a leap-frog scheme with a weak Asselin filter. The pressure perturbation term is solved using a Poisson solver.

For the purpose of using DNS to validate the analytical model (i.e. to confirm that no errors have been made in the derivation), it was necessary to run the DNS in a mode consistent with the assumptions of the analytical model. Accordingly, the coefficients $v$ and $\kappa$ are set to $10^{-4} \mathrm{~m}^{2} \mathrm{~s}^{-1}$, and we use $N=1 \mathrm{~s}^{-1}$. The latter value is not characteristic of the real atmosphere, observed values are of the order $10^{-2} \mathrm{~s}^{-1}$. However, for the purpose of validating the analytical model, which is a linear solution, the non-linear terms in the numerical simulation should be small (e.g. small Reynolds numbers), which is achieved by using a large $N$. The numerical simulation is further supplied with the slope angle $\alpha=3^{\circ}$, and the surface buoyancy of the strip $b_{0}=-0.01 \mathrm{~m} \mathrm{~s}^{-2}$. The scale factors introduced in the last section provide $l_{s}=4.37 \mathrm{~cm}, u_{s}=0.01 \mathrm{~m} \mathrm{~s}^{-1}$ and $b_{s}=0.01 \mathrm{~m} \mathrm{~s}^{-2}$, yielding the Reynolds number $\operatorname{Re}=\left(l_{s} u_{s}\right) / v \approx 4$.

In both the numerical simulation and analytical model, periodic boundary conditions are applied in the cross-slope direction. In order to simulate the flow induced by an isolated strip, a very large number of grid points is needed to put the lateral computational boundaries at a sufficiently large distance away from the cold strip (i.e. to have very large zones of neutrally buoyant surface on either side of the cold strip). The computational costs are reduced by considering a non-isolated strip; in this case the isolation parameter is $\mathcal{R}=3.41$. We adopted this value of $\mathcal{R}$ for the purpose of model verification.

The numerical simulation was carried out until a steady-state solution was reached. An excerpt of the DNS results is shown in Fig. 2 together with the analytical results.

Figure 2a shows the buoyancy and the downslope velocity at the centre of the strip. Only small differences are noted between the numerical and analytical results. The most pronounced discrepancy is in the downslope velocity. For $Z \lesssim 4$, the analytical model overestimates $U$, but at most by $\sim 10 \%$. The differences between the analytical and numerical results are likely due to a combined effect of the non-linear terms and numerical truncation errors.

In Fig. $2 b$ we show the numerical and analytical results for the buoyancy and the three velocity components as functions of cross-slope distance. All variables are evaluated at the height of the wind maximum $Z_{j}$. As noted above, the analytical model slightly overestimates the downslope velocity, foremost at $Y=0$. In the strip region, small differences are noted between the buoyancy profiles. The numerical results show a larger negative buoyancy. The largest relative difference between the analytical and numerical results of buoyancy is $3 \%$. Small differences are also seen in the profiles of cross-slope and slope-normal velocities. However, all variables point to a good overall agreement between the numerical and analytical data. 
Fig. 2 Comparison of numerical simulation and analytical results of laminar flow $(R e \approx 4)$ induced by a non-isolated strip: a buoyancy and downslope velocity at the centre of the strip, and $\mathbf{b}$ buoyancy and velocity components as functions of the cross-slope location. In $\mathbf{b}$ all variables are evaluated at the height of the wind maximum
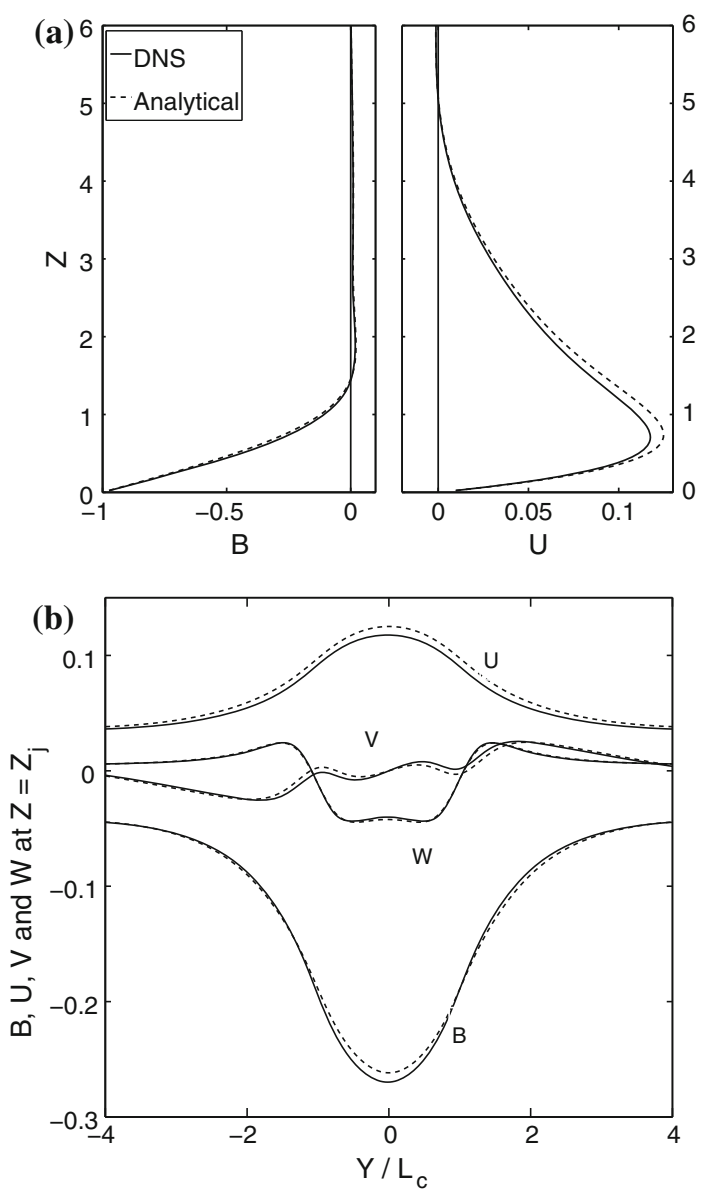

In the previous section, we discussed the application of periodic boundary conditions in the cross-slope direction. This implies that we consider repeated negatively buoyant strips separated by neutrally buoyant surface patches. In the remainder of this paper we focus on the flow induced by an isolated strip. A cold strip can be considered virtually isolated if the buoyancy and velocity components of the katabatic flow approach zero at a sufficiently large distance from the strip, which can only happen if the width of the neutrally buoyant surface is very large. In our model this is achieved by using a large $\mathcal{R}$. The question how large the isolation parameter should be in order for the strip to be regarded as isolated, is answered by running the analytical model with different $\mathcal{R}$ and looking for convergence. Relative differences between solutions to the model using $\mathcal{R}=250$ and 1000 were found to be negligible, hence $\mathcal{R}=250$ may be considered sufficient.

\section{Results}

In this section we analyze the influence of the strip width $L_{c}$ and slope angle $\alpha$ on the katabatic flow. We use the analytical model to evaluate three flow cases with $L_{c}=1,5$, and 10 whilst keeping the slope angle at $\alpha=5^{\circ}$, and three flow cases with $\alpha=1,5$, and $10^{\circ}$ 
keeping the strip width fixed at $L_{c}=5$. The input parameters are given in Table 1. Each case is assigned a label indicating the strip width and the slope angle, e.g. case L1A5 is with a strip width 1 and a slope angle of $5^{\circ}$.

Below we present flow variables over $Y-Z$ planes. The model variables are evaluated on a mesh with lateral and vertical grid spacing $\Delta Y=0.015$ and $\Delta Z=0.133$, respectively. The width of the domain is $-30 \leq Y / L_{c} \leq 30$ and its vertical extension is $0 \leq Z \leq 20$; for plotting purposes the figures only show a domain subsection. Furthermore, in the cross-slope direction the figures are scaled by the width of the strip in a manner that the surface cooling is always in the region $-1 \leq Y / L_{c} \leq 1$. Note that the lateral extent of the numerical mesh is not related to the isolation parameter.

\subsection{Buoyancy and downslope velocity}

The buoyancy in our model is a function of the cross-slope location as well as height. Figure 3 shows a cross-section of the buoyancy fields for various strip widths. At the surface, the scaled buoyancy is -1 in the strip region and 0 outside. For $Z \lesssim 2$ all cases show a pool of negative buoyancy (seen as an area enclosed by negative contour lines). The magnitude of this negative buoyancy drops sharply outside the strip region. Further aloft $B$ becomes positive as in the original Prandtl solution. The cross-slope and slope-normal extents of the positive buoyancy decrease with increasing $L_{c}$. Both the maximum and minimum of the buoyancy are found at the centre of the strip.

Figure 4 shows the buoyancy fields for various slope angles. The effect of the slope angle on the buoyancy field is similar to that of the strip width. With increasing $\alpha$, the cross-slope extents of the pools of both positive and negative $B$ decrease. Figure $4 \mathrm{a}-\mathrm{c}$ indicate that with increasing $\alpha$ the negative buoyancy field becomes increasingly homogeneous in the strip region.

Figure 5a, b display the vertical profiles of the mid-strip $(Y=0)$ buoyancy as a function of the strip width and slope angle, respectively. In all cases the buoyancy is -1 at the surface and increases to a (positive) maximum at $Z \sim 2-3.5$. The height and magnitude of the buoyancy

Table 1 Summary of modelled cases

\begin{tabular}{lllllllllr}
\hline & $L_{c}$ & $\alpha\left(^{\circ}\right)$ & $\begin{array}{l}\operatorname{Max}(B) \\
\left(10^{-2}\right)\end{array}$ & $Z_{\max (B)}$ & $\begin{array}{l}\operatorname{Max}(U) \\
\left(10^{-2}\right)\end{array}$ & $Z_{\max (U)}$ & $\begin{array}{l}\operatorname{Min}(U) \\
\left(10^{-2}\right)\end{array}$ & $Z_{\min (U)} Y_{\min (U)}$ \\
\hline L1A5 & 1 & 5 & 3.60 & 2.07 & 11.68 & 0.73 & -0.25 & 5.40 & 23.91 \\
L5A5 & 5 & 5 & 6.12 & 2.73 & 27.18 & 1.00 & -1.22 & 5.40 & 4.95 \\
L10A5 & 10 & 5 & 6.54 & 3.00 & 32.02 & 1.13 & -2.30 & 5.40 & 2.70 \\
L5A1 & 5 & 1 & 4.36 & 1.80 & 15.36 & 0.87 & -0.45 & 5.26 & 15.21 \\
L5A5 & 5 & 5 & 6.12 & 2.73 & 27.18 & 1.00 & -1.22 & 5.40 & 4.95 \\
L5A10 & 5 & 10 & 6.26 & 3.00 & 30.67 & 1.13 & -1.39 & 5.40 & 3.54 \\
Pr & $\infty$ & - & 6.70 & 3.33 & 32.24 & 1.11 & -1.39 & 5.55 & - \\
\hline
\end{tabular}

Rows are sorted according to strip width (rows 1-3) and slope angle (rows 4-6). Note that case L5A5 appears twice. Results from the Prandtl solution are shown in the bottom row. The maximum buoyancy max $(B)$ and downslope velocity $\max (U)$ are in the centre of the strip. The maximum counter flow is outside the strip, at height $Z_{\min (U)}$ and $Y= \pm Y_{\min (U)}$. In the dimensionless Prandtl solutions the extrema are independent of the slope angle, and there is no cross-slope variability in $B$ or $U$ 
Fig. 3 Contour plots of the buoyancy as a function of the strip width, illustrated by cases L1A5 (a) L5A5 (b) and L10A5 (c). Positive contour (black) intervals are 0.02 and negative contour (grey) intervals are 0.1 . Zero contours are indicated with " 0 ". Note that the horizontal axis is scaled to ensure that the buoyancy forcing is always between -1 and 1 . The $X$-axis is directed out of the plot
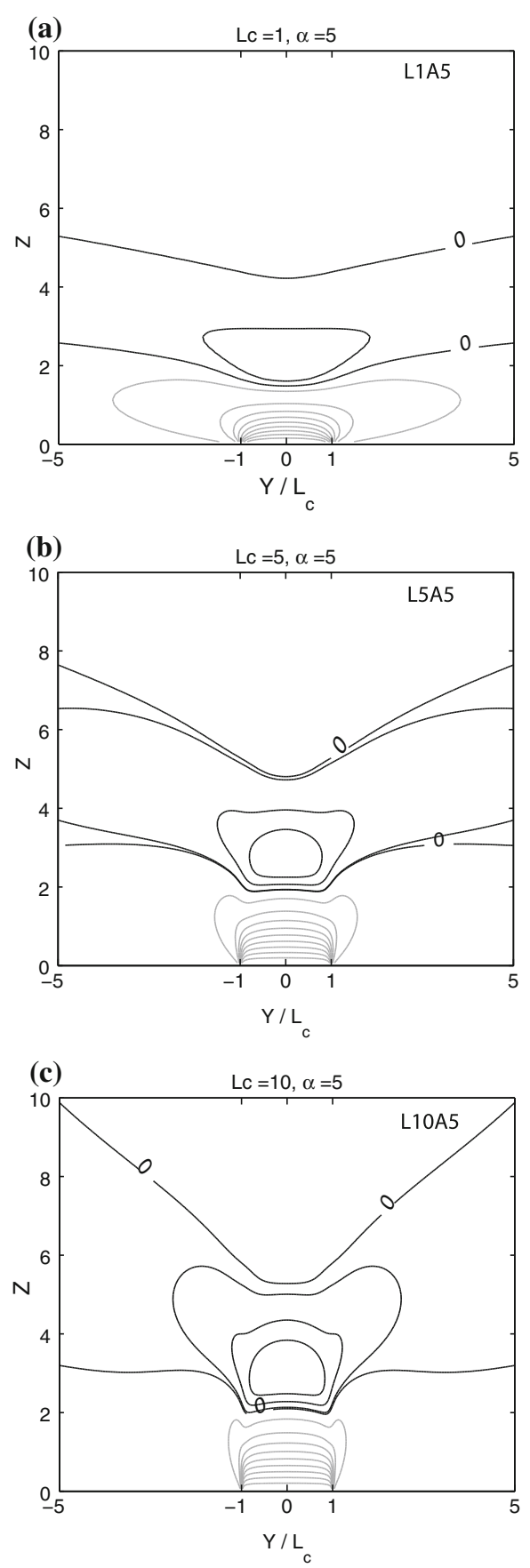

maximum increase with increasing $L_{c}$ and $\alpha$. For larger strip widths and slope angles, the buoyancy maximum height and magnitude approach values predicted by the Prandtl model.

Next we look at the downslope velocity $U$. Figure 6 presents contour plots of the downslope velocity as function of strip width. A jet-like wind maximum is observed over the 
Fig. 4 As in Fig. 3 but for the buoyancy as a function of the slope angle: a L5A1, b L5A5 and c L5A 10
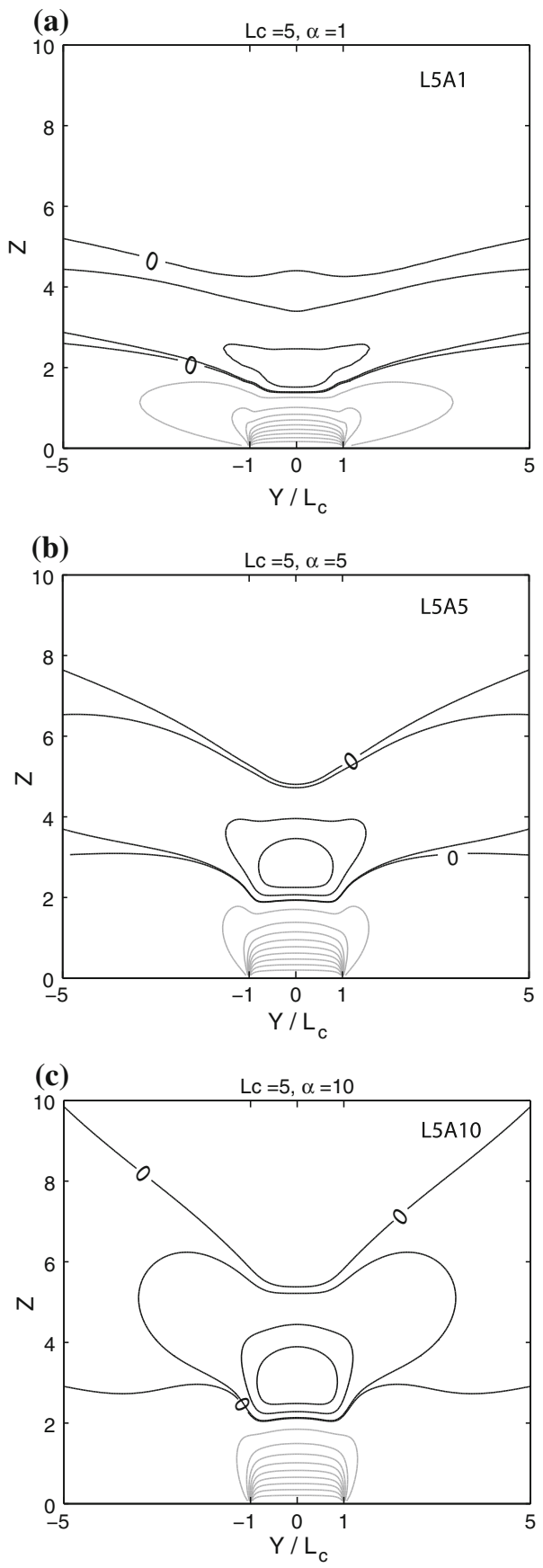

strip, and the downslope velocity spreads in the cross-slope direction. As $\alpha$ increases, the cross-slope spread of $U$ decreases but the vertical spread of $U$ increases.

There is a profound difference between the along-slope velocity field, $U$, predicted by our model and the Prandtl solutions. In the latter, the downslope wind maximum is capped 
Fig. 5 Vertical profiles of the buoyancy at the strip centre as functions of the a strip width and b slope angle. The dimensionless Prandtl profile of buoyancy (infinitely wide strip), is independent of the slope angle, included for comparison. See text for details

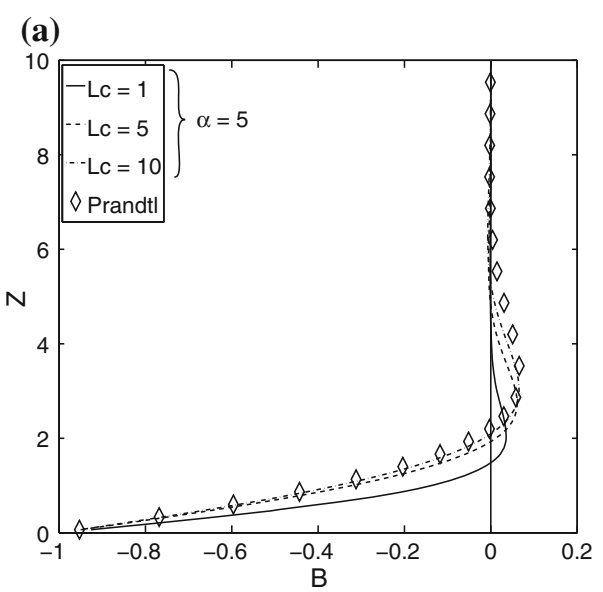

(b)

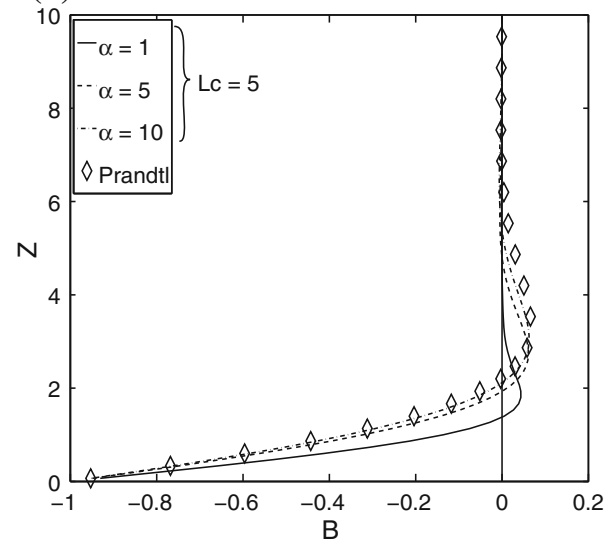

by a layer of weak up-slope flow. In our solution, however, the downslope velocity over the centre of the strip slowly approaches zero for large $Z$ without passing through negative values (no counter flow over the centre of the strip). Instead, the counter flow occurs outside the strip. The up-slope flow is symmetric in $Y=0$ as it takes place over the neutrally buoyant surfaces adjacent to the strip. A similar horizontal displacement of the return flow with respect to the strip was found in SF08, except the return flow there was not symmetric in $Y=0$ due to the Coriolis force.

For $L_{c}=1$, the counter flow is found at $|Y|>20$, which is outside the figure domain. Figure $6 \mathrm{~b}, \mathrm{c}$ and Table 1 show that with increasing $L_{c}$ the maximum counter flow intensifies and shifts closer to the strip. The height of the maximum counter flow $Z_{\min (U)}$ is fairly constant. Numerical results, evaluated on a grid with vertical grid distance $\Delta Z=0.133$, show that in all cases the height of the maximum counter flow is at $Z=5.40$. However, results from a case with a larger strip width $\left(L_{c}=15\right)$ show a maximum counter flow at $Z=5.53$, indicating that $Z_{\min (U)}$ varies only slowly $L_{c}$.

The contour plots of downslope velocity as function of the slope angle in Fig. 7 show several similarities with the downslope velocity fields as functions of the strip width in Fig. 6. For small slope angles the downslope flow extends in the cross-slope direction well beyond the strip. This extent decreases with increasing $\alpha$. Outside the strip region, the peak counter 
Fig. 6 As in Fig. 3 but for the downslope velocity $U$ as a function of the strip width. Positive contour interval is 0.025 , negative contour interval is 0.005 . Note that the positive flow is directed out of the paper
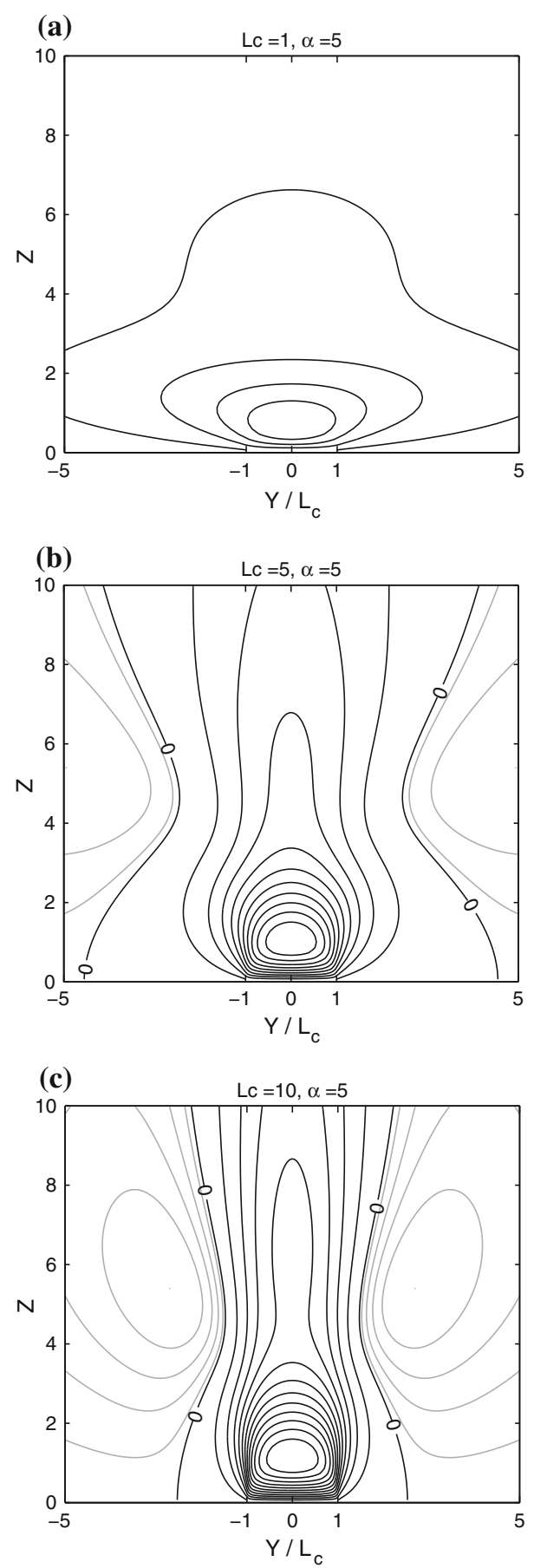

flow increases in magnitude and draws closer to the strip with increasing $\alpha$. The height of the maximum up-slope flow varies only slowly with $\alpha$, ranging from 5.26 to 5.40 (difference of one grid level). 
Fig. 7 As in Fig. 6 but for the downslope velocity $U$ as a function of the slope angle
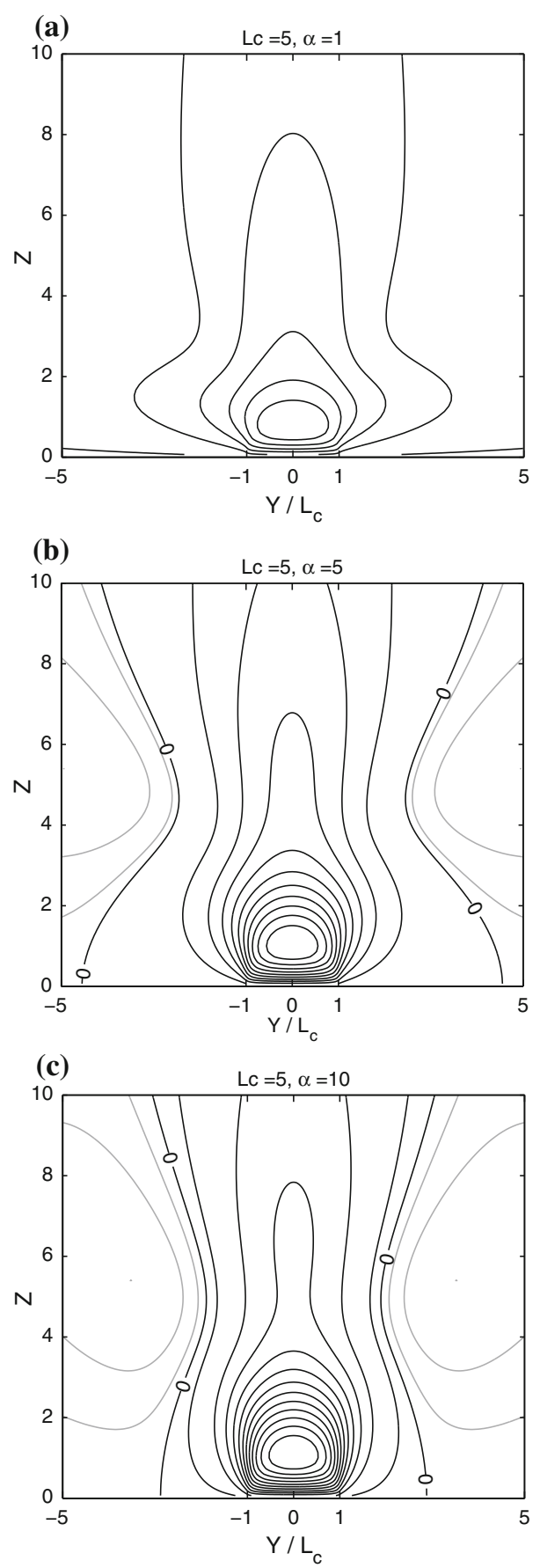

The wind maximum is located at the centre of the $\operatorname{strip}(Y=0)$ for all $L_{c}$ and $\alpha$. The vertical profiles of $U$ as a function of $L_{c}$ and $\alpha$ are shown in Fig. 8a, b, respectively. For the smallest strip width and slope angle, the wind maximum is less than half the wind maximum predicted 
Fig. 8 As Fig. 5 but for the downslope velocity $U$

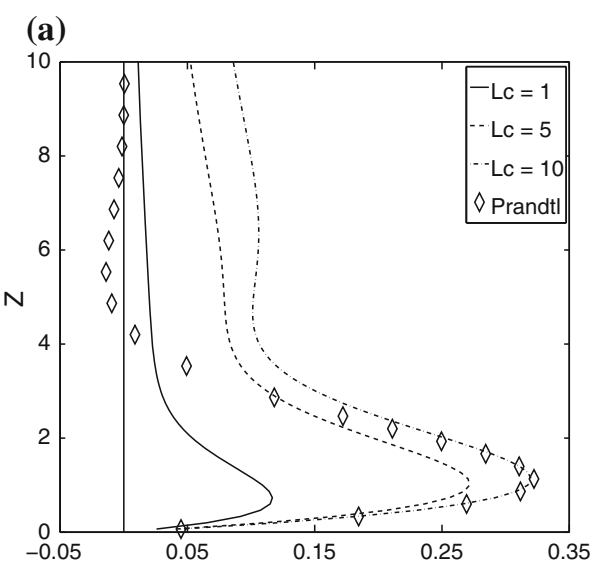

(b)

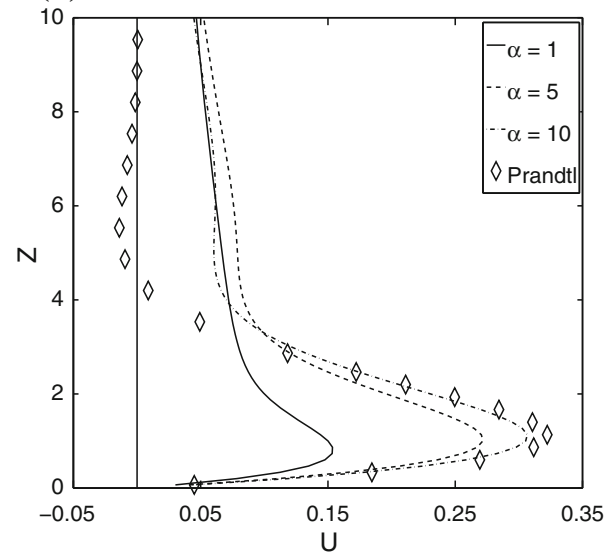

by the Prandtl model. The magnitude of the wind maximum increases with increasing $L_{c}$ and $\alpha$, and a small increase in the wind maximum height $Z_{j}$ is observed. It is interesting to point out that in the dimensionless Prandtl solutions, the height and magnitude of the wind maximum are independent of the slope angle.

A notable characteristic of the Prandtl solutions is the velocity oscillation about zero as $Z$ increases. With a limited strip width the oscillation is the most pronounced for the wide strip and steep slope. However, the oscillation occurs around a positive value of $U$. All velocity profiles at the centre of the strip tend to zero for large $Z$ without passing through negative values.

\subsection{Cross-slope and slope-normal flow}

The inhomogeneous surface forcing in our model produces vertical and cross-slope motions. Figure 9 shows contour plots of the stream function $\Psi$ as a function of $L_{c}$. Close to the surface, vortices near the strip edges are observed. The motion is clockwise along negative contours. Below the wind maximum, the fluid above the cold surface descends and moves towards the strip edge where it is detrained from the strip region. Outside the strip region, the motion is at first away from the strip and ascending. There after a rising motion towards 
the strip is observed. The fluid re-enters the strip region above the wind maximum, and the circulation is closed by a branch of sinking motion.

Near the surface, $Z \lesssim 2.5$, the density of the contour lines increase with increasing $L_{c}$. This density increase corresponds to the increase in magnitudes of $V$ and $W$. Figure 9 and Table 2 show that the height of the maximum $\Psi$ is close to the height of the downslope wind maximum $Z_{j}$. The cross-slope circulation in the interior of the strip region $\left(\left|Y / L_{c}\right| \lesssim 0.5\right.$, $Z \lesssim 2.5$ ) becomes centered around the strip edges for an increasing strip width. For large $L_{c}$, our model solution near the strip centre resembles the Prandtl solutions.

Farther away from the surface, $Z \gtrsim 2.5$, another set of vortices is observed. The circulation is opposite to the one in the lowermost vortices. We note that the cross-slope extent of the elevated pair of vortices decreases with increasing $L_{c}$. Instead, the vertical size of the vortices increases. For cases L1A5 and L5A5 the maximum $\Psi$ is found near the wind maximum height, but for larger $L_{c}$, e.g. in the L10A5 case, $\max (\Psi)$ is associated with the uppermost pair of vortices $(Z \sim 5.75)$.

Figure 10 shows $\Psi$ as a function of the slope angle. An inspection of Figs. 9 and 10 allows to conclude that an increasing slope angle affects $\Psi$ similarly to an increase in $L_{c}$. In Fig. 10, the maximum $\Psi$ at $Z \gtrsim 2.5$ increases with increasing $\alpha$, and the cross-slope size of the vortices decreases whereas the vertical size increases. A notable difference with Fig. 9 is that the maximum $\Psi$ close to the surface $(Z \lesssim 2.5)$ at some point starts decreasing. Near the surface, the maximum values of $\Psi$ for $\alpha=5$ and $10^{\circ}$ are $8.4 \times 10^{-2}$ and $7.0 \times 10^{-2}$, respectively. The maximum $V$ values for cases L5A5 and L5A10 are, respectively, $3.6 \times 10^{-2}$ and $3.2 \times 10^{-2}$. As may be concluded from the gradients of $\Psi$, the cross-slope velocity and slope-normal velocity both decrease in magnitude as $\alpha$ increases. Apparently, for slope angles larger than some critical value, $\alpha>\alpha_{c}$, the cross-slope circulation decreases. Since the cross-slope circulation is also determined by the strip width, we expect $\alpha_{c}$ to be a function of $L_{c}$ as well. The reason for this issue is still unknown, and should be studied further.

\section{A dimensional framework}

The previous section describes the structure of the flow. We found the cross-slope extent of the buoyancy field perturbation to decrease with increasing slope angle. In a dimensional framework, this effect can be explained by considering the steady-state, linearized (with respect to the downslope coordinate) buoyancy Eq. 1 and downslope momentum Eq. 2 at the edge of the strip. In this region, the $y$-derivatives in the Laplacian can be expected to be of the same order of magnitude (or larger) than the $z$-derivative terms. Accordingly, the downslope advection of environmental potential temperature is of the same order of magnitude as the cross-slope diffusion, i.e.

$$
N^{2} u \sin \alpha \sim \kappa \frac{\partial^{2} b}{\partial y^{2}} .
$$

Introducing an (unspecified) horizontal length scale $\tilde{L}$, velocity scale $\tilde{u}$ and buoyancy scale $\tilde{b}$ in the above relation yields

$$
\tilde{u} N^{2} \sin \alpha \sim \kappa \tilde{b} / \tilde{L}^{2} .
$$

An analogous scale transformation of the steady-state linearized homogeneous downslope equation of motion (2) near the strip edge yields

$$
\tilde{b} \sin \alpha \sim \nu \tilde{u} / \tilde{L}^{2} .
$$


Fig. 9 As Fig. 3 but for the stream function $\Psi$ as a function of $L_{c}$. The motion is clockwise along negative contours. Positive and negative contour intervals are 0.02
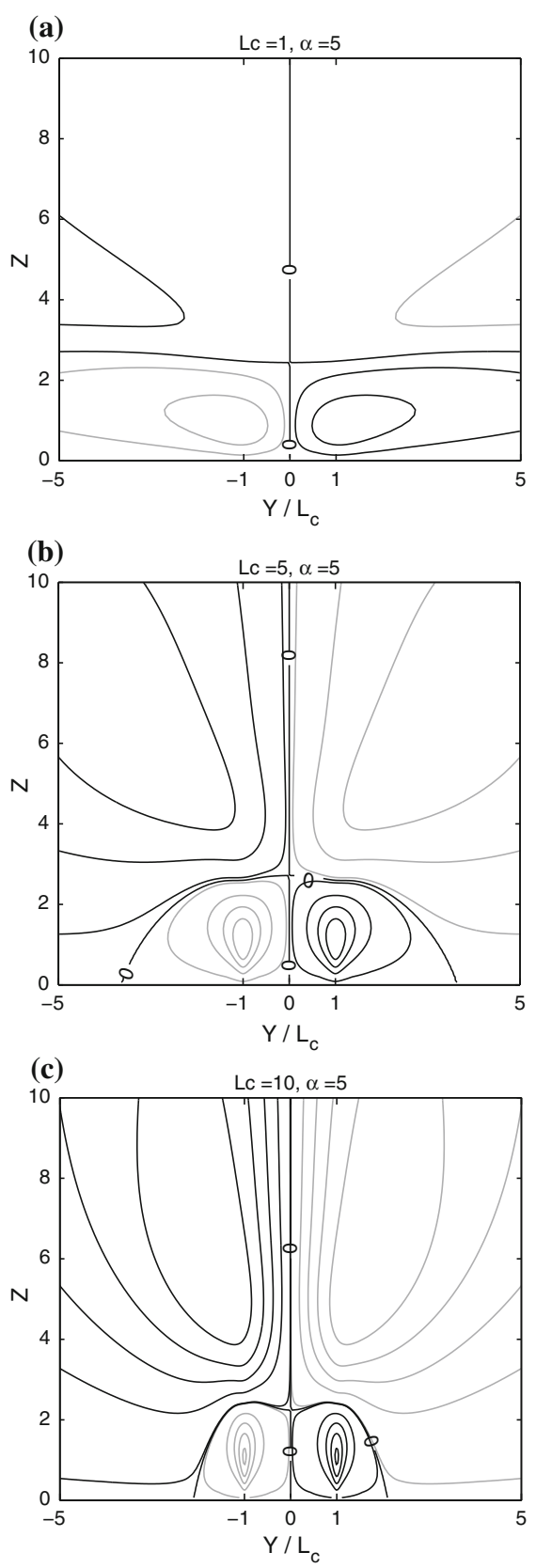

Note that neither of these two scaling relations by itself provides a formula for $\tilde{u}, \tilde{b}$ or $\tilde{L}$ in terms of the governing parameters. However, combined they yield a formula for the horizontal length scale

$$
\tilde{L} \sim \frac{(\kappa \nu)^{1 / 4}}{\sqrt{N \sin \alpha}} .
$$


Table 2 Summary of maxima in the stream function $\Psi$ in terms of absolute values of $\Psi$

\begin{tabular}{lllllll}
\hline & $\operatorname{Max}(\Psi)\left(10^{-2}\right)$ & $Y_{\max (\Psi)}$ & $Z_{\max (\Psi)}$ & $\operatorname{Max}(\Psi)\left(10^{-2}\right)$ & $Y_{\max (\Psi)}$ & $Z_{\max (\Psi)}$ \\
\hline L1A5 & 4.68 & 1.20 & 0.87 & 0.01 & 11.28 & 5.26 \\
L5A5 & 8.37 & 0.99 & 1.13 & 5.39 & 2.55 & 5.40 \\
L10A5 & 8.44 & 0.99 & 1.13 & 10.07 & 1.59 & 5.67 \\
L5A1 & 7.22 & 1.02 & 0.73 & 1.59 & 6.60 & 4.73 \\
L5A5 & 8.37 & 0.99 & 1.13 & 5.39 & 2.55 & 5.40 \\
L5A10 & 7.03 & 0.99 & 1.27 & 7.42 & 1.98 & 5.80 \\
& $Z \leq 2.5$ & & & $Z>2.5$ & & \\
\hline
\end{tabular}

Note that $\Psi$ is an odd function of $Y$, so that the minimum values in $\Psi$ are equal in magnitude to the maximum values. The first three columns include data on the pair of vortices close to the surface $(Z \lesssim 2.5)$ whereas the three last columns refer to the elevated pair of vortices $(Z \gtrsim 2.5)$

This relation for the horizontal length scale is analogous to the relation for the vertical length scale in the classical Prandtl solution. It shows that the horizontal length scale decreases as the slope angle increases, which is in qualitative agreement with the results from the complete analytical solution. Note that the cross-slope axis in the figures is plotted as $Y / L_{c}$ which is itself a ratio of two terms which have both been non-dimensionalized with $l_{s}$. This ratio is actually equivalent to the ratio of the dimensional $y$ to the dimensional half-strip width. The lateral scale dependence on the slope angle can therefore be immediately (visually) inferred from the contour plots. In contrast, as discussed below, care must be taken in inferring the vertical scale dependencies from the contour plots.

The strength of the dimensionless analytical solution is that it represents all possible dimensional results. On the other hand, the scaling of the variables does to some degree mask the results. For example, we previously found the dimensionless height of the buoyancy maximum to increase with increasing $\alpha$. A dimensional height can be recovered by using the scale height $l_{s}$, which is inverse proportional to the slope angle, e.g. $l_{s} \propto(\sin \alpha)^{-1 / 2}$. In a dimensional framework, the height of the maximum buoyancy therefore decreases with increasing $\alpha$. This decrease by far exceeds the increasing trend that we found in the dimensionless framework.

We also found the non-dimensional height of the wind maximum to increase with increasing $\alpha$, and that this height approaches the (dimensionless) Prandtl prediction for steep slopes. In a dimensional framework, however, the Prandtl solution shows that the wind maximum height occurs at progressively decreasing heights for increasing slope angles. Our analytical model shows similar results when expressed in a dimensional form.

The introduction of $l_{s}$ as a scale factor for the cross-slope distance also affects how the results concerning the dimensionless loci of the up-slope wind maxima should be interpreted. Although these maxima shift towards the strip for larger $\alpha$, in a dimensional framework the shift will be more rapid due to the effect of $\alpha$ on $l_{s}$.

\section{Conclusions}

In this paper we focus on small-scale shallow katabatic flow for which the Coriolis effect is assumed to be negligible. The flow is induced by a negatively buoyant strip of finite width. We present a dimensionless analytical solution in which the slope angle and the (dimensionless) width of the negatively buoyant strip are governing parameters. 
Fig. 10 As Fig. 9 but as a function of the slope angle
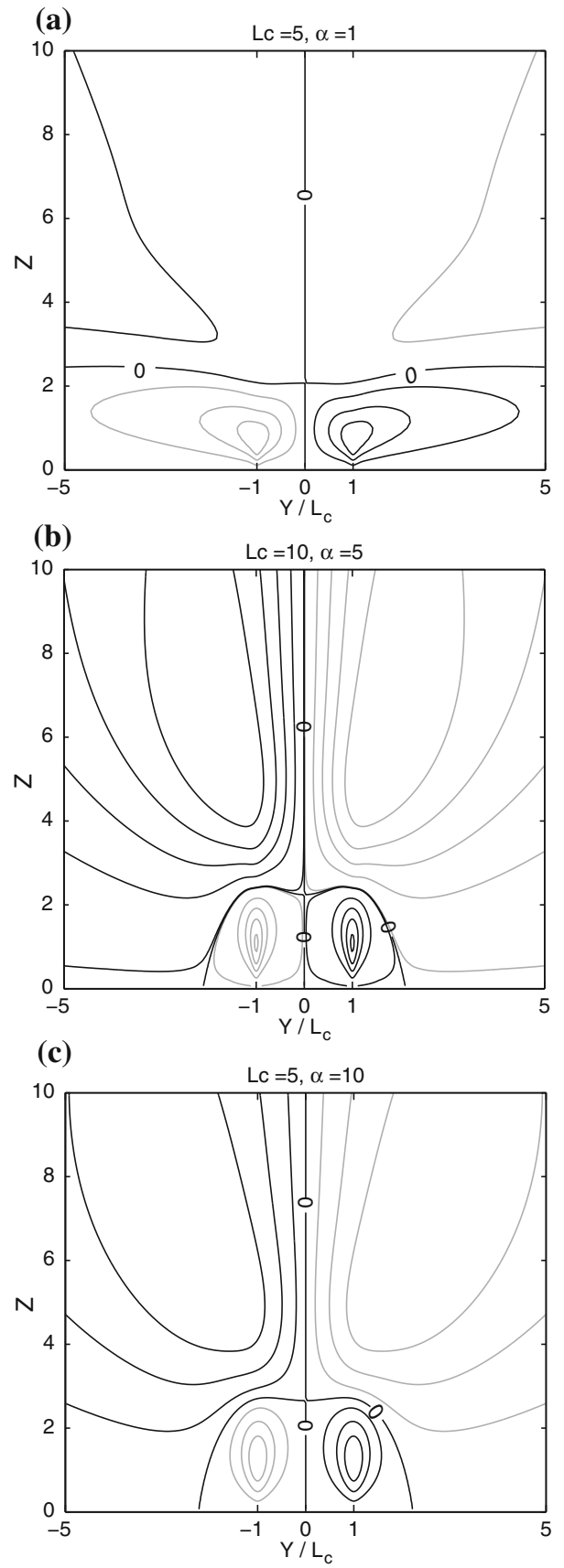

The developed analytical model can be viewed as an extension to the Prandtl model. We have verified that the model reverts to the classical Prandtl model for an infinitely wide strip. The model is also validated by comparing the results to a DNS of a katabatic flow with a low Reynolds number $(R e \approx 4)$. 
The effects of the strip width $L_{c}$ and the slope angle $\alpha$ are investigated. The negative buoyancy diffuses from the strip primarily outward. The outward diffusion is smaller for broader strips and larger slope angles. At the centre of the strip, the maximum (positive) buoyancy increases for broad strips and large slope angles. A limit for the maximum buoyancy is given by the Prandtl model. It is interesting to note that observations of glacier winds hardly show any positive buoyancy values [27]. Compared to the Prandtl model, the reduced positive buoyancy predicted by our model is thus in better qualitative agreement with observations. This suggests that secondary lateral circulations, which are likely to occur in a katabatic flow of finite width, may be important for the katabatic flow dynamics.

The downslope velocity, which is caused by the negative buoyancy, attains a maximum at the centre of the strip. The magnitude of the maximum decreases toward the edges of the strip. As in the Prandtl model, we observe a counter flow. A particular result from our model, though, is that the counter flow is located outside the strip region. For larger $L_{c}$ and $\alpha$, the positive downslope velocity field becomes more confined to the strip region, while the counter flow intensifies and moves toward the strip. With increasing $L_{c}$ and $\alpha$, the magnitude of the wind maximum approaches the wind maximum predicted by the Prandtl model.

In the non-dimensional Prandtl solution, the downslope wind decreases rapidly above the wind maximum and then oscillates around $U=0$, as shown in Fig. 8. In the case of an isolated strip, however, the downslope velocity at the centre of the strip remains positive and reduces to zero monotonically. The relatively slow decrease in $U$ above the wind maximum is supported by observations of katabatic flow over valley glacier, see e.g. [27], which allows to conclude that the glacier wind observed over the Pasterze glacier, Austria, had a positive downslope velocity up to 50-200 m. The same observations, reanalyzed in [28], showed that the height at which the downslope velocity became negative, had a diurnal cycle. It was also found that the daytime heating of the (ice-free) valley walls induced a downslope flow that merged with the glacier wind. These findings again illustrate the importance of including a cross-slope variation in the surface buoyancy when modelling the katabatic flow over a valley glacier.

In our analytical model, the finiteness of the negatively buoyant strip is responsible for inducing a cross-slope circulation. As in SF08, we find that close to the surface, a vortex develops at each strip edge. Immediately above the surface, fluid descends and is detrained from the strip region. Outside the strip, the fluid first attains an outward ascending motion where after the direction shifts towards the strip. Upon re-entering the strip region the fluid starts descending again.

Farther aloft, a second pair of counter-rotating vortices produce an ascending and outward motion above the strip. The intensity of the two pairs of vortices increases with increasing $L_{c}$ and $\alpha$. By increasing the slope angle from $5^{\circ}$ to $10^{\circ}$, we found that the circulation in the lowermost pair of vortices decreases. This suggests that the intensity of the cross-slope and slope-normal flow has a maximum at some given slope angle.

An apparent deficiency of the analytical model is the neglect of the non-linear terms in the governing equations. For low Reynolds number laminar flow, this approach does work as was shown by comparison of analytical predictions with DNS results of a non-turbulent flow. However, for large Reynolds numbers (turbulent flow) we cannot a priori assume a negligible cross-slope advection of e.g. cross-slope velocity. Preliminary results from a large-eddy simulation, described in [27], of turbulent katabatic flow over a non-islated strip $(\mathcal{R}=4)$ has shown that below the wind maximum the turbulent diffusion terms in the momentum balance are larger than the advection terms. However, farther aloft, turbulent diffusion plays only a minor role. The large-eddy simulation results do show a near-surface vortex at each strip edge, but unlike the analytical model no elevated vortices are found. Future tests should 
be conducted to see if the elevated vortices appear in large-eddy simulations for flow cases where the strip is more isolated.

Turbulent flows are frequently modelled using closure theory, which introduces turbulence exchange coefficients. The exchange coefficient for momentum is commonly taken proportional to the wind shear, and thus vanish at the height of the wind maximum. Such a height-dependency has not been included in our model, and may as such be regarded as a deficiency of the analytical solution. On the other hand, we in fact solve a laminar flow problem where the exchange coefficients (diffusivity coefficients) are constant. This does to some degree diminish the applicability of the analytical model to the real atmosphere, and it must therefore be appreciated as a conceptual model.

In the proposed analytical model, the surface buoyancy was assumed to be symmetric around the strip centre. A cross-slope variation of the surface buoyancy different from the one discussed in this paper may be considered, provided the requirement of symmetry is fulfilled. However, a symmetric surface forcing is a highly idealized situation. For instance, diurnal solar heating of the air above a valley glacier is usually asymmetric. A possible extension of our model would be able to account for such an asymmetric surface forcing.

Acknowledgements A. Shapiro and E. Fedorovich gratefully acknowledge support from the National Science Foundation, USA, through the grant ATM-0622745.

Open Access This article is distributed under the terms of the Creative Commons Attribution Noncommercial License which permits any noncommercial use, distribution, and reproduction in any medium, provided the original author(s) and source are credited.

\section{Appendix A: general solution}

Applying the Fourier representation (16) in (14) yields:

$$
\begin{aligned}
0= & \sum_{n=1}^{\infty} \cos (p Y)\left[\frac{\mathrm{d}^{6} a_{n}}{\mathrm{~d} Z^{6}}-3 p^{2} \frac{\mathrm{d}^{4} a_{n}}{\mathrm{~d} Z^{4}}+\left(3 p^{4}+1\right) \frac{\mathrm{d}^{2} a_{n}}{\mathrm{~d} Z^{2}}-p^{6} a_{n}-p^{2}(c+1) a_{n}\right] \\
& +a_{0}(Z) .
\end{aligned}
$$

Below it will be apparent that the $n=0$ contribution to the Fourier series will yield a singularity. Therefore we first present a solution for $n>0$. The $n=0$ contribution is analyzed separately in Appendix C.

Assume that $a_{n}$ behaves exponentially: $a_{n}(Z) \propto e^{m Z}$, with proportionality coefficients being determined by the boundary conditions. Since the $\cos (p Y)$ factors (as functions of $n$ ) are linearly independent, the terms enclosed by square brackets should sum to zero for every $n$, hence

$$
0=\xi^{3}-3 t \xi^{2}+\left(3 t^{2}+1\right) \xi-t^{3}-t c-t
$$

where $\xi \equiv m^{2}$ and $t \equiv p^{2}$.

The general solution to the cubic equation $\chi^{3}+h_{2} \chi^{2}+h_{1} \chi+h_{0}=0\left(h_{i}\right.$ are constants $)$ is [29]: 


$$
\begin{aligned}
& \chi_{1}=\frac{-1}{3} h_{2}+(S+T), \\
& \chi_{2}, \chi_{3}=\frac{-1}{3} h_{2}-\frac{1}{2}(S+T) \pm \frac{i \sqrt{3}}{2}(S-T), \\
& S=[R+\sqrt{D}]^{1 / 3}, \quad T=[R-\sqrt{D}]^{1 / 3} \text { with } D=Q^{3}+R^{2}, \\
& Q=\frac{3 h_{1}-h_{2}^{2}}{9} \text { and } R=\frac{9 h_{2} h_{1}-27 h_{0}-2 h_{2}^{3}}{54} .
\end{aligned}
$$

The roots of $\xi$ become

$$
\xi_{1}=t+\rho, \quad \xi_{2}=t-\frac{1}{2} \rho+\frac{i \sqrt{3}}{2} \eta, \quad \text { and } \quad \xi_{3}=t-\frac{1}{2} \rho-\frac{i \sqrt{3}}{2} \eta,
$$

where $\rho \equiv \mathcal{S}^{1 / 3}-\mathcal{T}^{1 / 3}, \eta \equiv \mathcal{S}^{1 / 3}+\mathcal{T}^{1 / 3}$ and

$$
\mathcal{S} \equiv \frac{t c}{2}+\sqrt{\frac{1}{27}+\frac{t^{2} c^{2}}{4}} \text { and } \mathcal{T} \equiv\left|\frac{t c}{2}-\sqrt{\frac{1}{27}+\frac{t^{2} c^{2}}{4}}\right| .
$$

The polar form of the complex root $\xi_{2}$ can be written as

$$
\xi_{2}=\sqrt{(t-\rho / 2+i \sqrt{3} \eta / 2)(t-\rho / 2-i \sqrt{3} \eta / 2)} e^{i \theta}=\zeta e^{i \theta},
$$

and similarly $\xi_{3}=\zeta e^{-i \theta}$, where $\theta=\operatorname{atan}[(\sqrt{3} \eta / 2) /(t-\rho / 2)]$.

More precisely,

$$
\begin{aligned}
& \zeta \cos \theta=t-\rho / 2 \Rightarrow \cos \theta=\frac{t-\rho / 2}{\zeta}, \\
& \zeta \sin \theta=\sqrt{3} \eta / 2 \Rightarrow \sin \theta=\frac{\sqrt{3}}{2} \frac{\eta}{\zeta}
\end{aligned}
$$

Since both $\eta$ and $\zeta$ are positive, $\sin \theta$ is positive, which implies that $\theta$ is in the first or second quadrant, depending on the sign of $t-\rho / 2$. Therefore, when obtaining the roots $m= \pm \sqrt{\xi_{2}}$, the angle $\theta / 2$ is guaranteed to be in the first quadrant. We seek for $a_{n}$ in the form of exponential functions $e^{m Z}$ that are bounded for all $Z$, and thus the roots with positive real parts must be rejected.

The three physical roots are

$$
m_{1}=-\sqrt{t+\rho} \quad, \quad m_{2}=-\sqrt{\zeta} e^{i \theta / 2} \text { and } m_{3}=-\sqrt{\zeta} e^{-i \theta / 2} .
$$

The expression for $a_{n}$ is a superposition of the three exponential functions:

$$
a_{n}(Z)=C_{n} e^{m_{1} Z}+D_{n} e^{m_{2} Z}+E_{n} e^{m_{3} Z},
$$

and the full solution to (A.1) becomes

$$
B(Y, Z)=\sum_{n=1}^{\infty} \cos (p Y)\left[C_{n} e^{m_{1} Z}+D_{n} e^{m_{2} Z}+E_{n} e^{m_{3} Z}\right],
$$

with $C_{n}, D_{n}$ and $E_{n}$ being constants determined by the boundary conditions. 
The stream function $\Psi$ is constrained by (13) to satisfy

$$
\nabla^{4} \Psi=\cot \alpha \sum_{n=1}^{\infty}-p \sin (p Y)\left[C_{n} e^{m_{1} Z}+D_{n} e^{m_{2} Z}+E_{n} e^{m_{3} Z}\right],
$$

which is a linear, non-homogeneous differential equation. The solution is the sum of the solution to the homogeneous differential equation plus a particular solution, $\Psi=\Psi_{h}+\Psi_{p}$. Seeking a particular solution on the form

$$
\Psi_{p}=\sum_{n=1}^{\infty} \sin (p Y)\left[\beta_{1, n} C_{n} e^{m_{1} Z}+\beta_{2, n} D_{n} e^{m_{2} Z}+\beta_{3, n} E_{n} e^{m_{3} Z}\right],
$$

where $\beta_{\sigma, n}$ are constants, we get

$$
\begin{aligned}
\nabla^{4} \Psi_{p} & =\sum_{n=1}^{\infty} \sin (p Y)\left\{\beta_{1, n} C_{n}\left(m_{1}^{4}-2 p^{2} m_{1}^{2}+p^{4}\right) e^{m_{1} Z}\right. \\
& \left.+\beta_{2, n} D_{n}\left(m_{2}^{4}-2 p^{2} m_{2}^{2}+p^{4}\right) e^{m_{2} Z}+\beta_{3, n} E_{n}\left(m_{3}^{4}-2 p^{2} m_{3}^{2}+p^{4}\right) e^{m_{3} Z}\right\} .
\end{aligned}
$$

Applying (A.5) in (A.4) yields

$$
\begin{aligned}
& \beta_{1, n}=-p \cot \alpha /\left(m_{1}^{4}-2 p^{2} m_{1}^{2}+p^{4}\right), \\
& \beta_{2, n}=-p \cot \alpha /\left(m_{2}^{4}-2 p^{2} m_{2}^{2}+p^{4}\right), \\
& \beta_{3, n}=-p \cot \alpha /\left(m_{3}^{4}-2 p^{2} m_{3}^{2}+p^{4}\right) .
\end{aligned}
$$

In solving the homogeneous part of the differential equation, we assume $\Psi_{h}=\sum_{n=1}^{\infty} \tilde{\psi}_{n} \sin$ $(p Y)$ and $\tilde{\psi}_{n}=\gamma_{n} e^{g Z}, \gamma_{n}$ being constants, yielding

$$
\nabla^{4} \Psi_{h}=\sum_{n=1}^{\infty}\left(g^{4}-2 g^{2} p^{2}+p^{4}\right) \gamma_{n} e^{g z} \sin (p Y)=0 .
$$

Since $\sin (p Y)$ are linearly independent (for different $n$ ), we must have $g^{4}-2 g^{2} p^{2}+p^{4}=0$, or $g= \pm p$. A fourth-order differential equation has four solutions. In this case $\tilde{\psi}$ must be of the form $\gamma_{n} e^{ \pm p Z}$ or $\gamma_{n} Z e^{ \pm p Z}$ [30]. Since the solutions must be bounded as $Z$ goes to infinity, the terms associated with a positive exponent are omitted. Moreover, it can be shown that the expressions for $U$ and $B$ do not contain terms on the form $Z e^{-p Z}$, i.e. they must also be rejected from the solution to the homogeneous differential equation. We thus find that

$$
\Psi_{h}=\sum_{n=1}^{\infty} \gamma_{n} e^{-p Z} \sin (p Y)
$$

The full solution to $\Psi$ becomes

$$
\Psi=\sum_{n=1}^{\infty} \sin (p Y)\left[\beta_{1, n} C_{n} e^{m_{1} Z}+\beta_{2, n} D_{n} e^{m_{2} Z}+\beta_{3, n} E_{n} e^{m_{3} Z}+\gamma_{n} e^{-p Z}\right] .
$$

From Eq. 12 we have $\nabla^{2} U=B$, which is a second-order non-homogeneous differential equation. Following the approach above, we will solve for $U=U_{h}+U_{p}$ where we seek the particular solution $U_{p}$ in the form 


$$
U_{p}=\sum_{n=1}^{\infty} \cos (p Y)\left[\delta_{1, n} C_{n} e^{m_{1} Z}+\delta_{2, n} D_{n} e^{m_{2} Z}+\delta_{3, n} E_{n} e^{m_{3} Z}\right],
$$

with $\delta_{\sigma, n}$ being constants. The Laplacian of the particular solution is:

$$
\begin{aligned}
\nabla^{2} U_{p} & =\sum_{n=1}^{\infty} \cos (p Y)\left[\left(m_{1}^{2}-p^{2}\right) \delta_{1, n} C_{n} e^{m_{1} Z}\right. \\
& \left.+\left(m_{2}^{2}-p^{2}\right) \delta_{2, n} D_{n} e^{m_{1} Z}+\left(m_{3}^{2}-p^{2}\right) \delta_{3, n} E_{n} e^{m_{1} Z}\right]
\end{aligned}
$$

which must equal the right hand side of (A.3). Equating exponents of the same power yields:

$$
\delta_{1, n}=1 /\left(m_{1}^{2}-p^{2}\right), \quad \delta_{2, n}=1 /\left(m_{2}^{2}-p^{2}\right), \quad \delta_{3, n}=1 /\left(m_{3}^{2}-p^{2}\right) .
$$

For the homogeneous part $U_{h}$, assume a solution on the form $U_{h}=\sum_{n=1}^{\infty} \tilde{u} \cos (p Y)$ with $\tilde{u}=\epsilon_{n} e^{g Z}$, where $\epsilon_{n}$ are constants. The Laplacian of $U_{h}$ gives

$$
\nabla^{2} U_{h}=\sum_{n=1}^{\infty} \cos (p Y)\left(g^{2}-p^{2}\right) \epsilon_{n} e^{g Z} .
$$

Since the right hand side of (A.11) must vanish, $g= \pm p$. Omitting the non-physical root $g=+p$, the sum of the homogeneous and particular solutions becomes

$$
U=\sum_{n=1}^{\infty} \cos (p Y)\left[\delta_{1, n} C_{n} e^{m_{1} Z}+\delta_{2, n} D_{n} e^{m_{2} Z}+\delta_{3, n} E_{n} e^{m_{3} Z}+\epsilon_{n} e^{-p Z}\right] .
$$

This concludes the general solution to the three variables $B, \Psi$ and $U$ given by (A.3), (A.8) and (A.12), respectively, with constants given by (A.6) and (A.10).

\section{Appendix B: surface boundary conditions}

In deriving the general expressions for $B, U$ and $\Psi$ we applied the periodic boundary condition in the cross-slope direction, and required that flow variables vanish as $Z \rightarrow \infty$. Here we focus on the surface boundary conditions $(Z=0)$.

The surface buoyancy is -1 in the strip region and 0 outside, i.e.

$$
B_{S}(Y) \equiv B(Y, 0)=-1 \quad \text { if }|Y| \leq L_{c}, \quad 0 \text { otherwise. }
$$

The Fourier representation is

$$
B_{s}=\sum_{n=1}^{\infty} \mathcal{B}_{n} \cos (p Y)+\mathcal{B}_{0} .
$$

The coefficients $\mathcal{B}_{n}$ are given by

$$
\mathcal{B}_{0}=\frac{1}{\tau} \int_{-\tau / 2}^{\tau / 2} B_{s} d Y=\frac{-1}{1+\mathcal{R}}
$$

and

$$
\mathcal{B}_{n}=\frac{2}{\tau} \int_{-\tau / 2}^{\tau / 2} B_{s} \cos (p Y) d Y=\frac{-2}{n \pi} \sin \left(\frac{n \pi}{1+\mathcal{R}}\right) .
$$


Combining Eqs. A.3 and B.1 evaluated at the surface gives

$$
\mathcal{B}_{n}=C_{n}+D_{n}+E_{n} .
$$

At the surface we further impose the no-slip condition for the three velocity components. For $n>0$, evaluating Eqs. A.8 and A.12 yields for $U, V=\partial \Psi / \partial Z$, and $W=-\partial \Psi / \partial Y$, at $Z=0$ yields, respectively

$$
\begin{aligned}
& 0=\delta_{1, n} C_{n}+\delta_{2, n} D_{n}+\delta_{3, n} E_{n}+\epsilon_{n}, \\
& 0=m_{1} \beta_{1, n} C_{n}+m_{2} \beta_{2, n} D_{n}+m_{3} \beta_{3, n} E_{n}-p \gamma_{n}, \\
& 0=-\beta_{1, n} C_{n}-\beta_{2, n} D_{n}-\beta_{3, n} E_{n}-\gamma_{n} .
\end{aligned}
$$

Lastly, using the Fourier representation of $U, \Psi$ and $B$ in (11) at $Z=0$, we obtain

$$
\begin{aligned}
0= & \sum_{n=1}^{\infty} \cos (p Y)\left\{C_{n}\left(\delta_{1, n}+p \beta_{1, n} \sqrt{c}+m_{1}^{2}-p^{2}\right)+D_{n}\left(\delta_{2, n}+p \beta_{2, n} \sqrt{c}+m_{2}^{2}-p^{2}\right)\right. \\
& \left.+E_{n}\left(\delta_{3, n}+p \beta_{3, n} \sqrt{c}+m_{3}^{2}-p^{2}\right)+\left(\epsilon_{n}+p \sqrt{c} \gamma_{n}\right)\right\} .
\end{aligned}
$$

In Eq. 21 the surface boundary conditions are summarized in a matrix form.

\section{Appendix C: the $\boldsymbol{n}=\mathbf{0}$ contribution and the Prandtl solution}

In the analytical model developed, the constants $\beta_{\sigma, n}$ and $\delta_{\sigma, n}$ in (A.6) and (A.10), respectively, become singular for $n=0$. The first term in the Fourier representations of $B$ and $U$ must therefore be treated separately. Note that since the stream function is proportional to $\sin (p Y)$, Eq. A. 8 has no $n=0$ contribution.

For $n=0$, Eq. A. 1 reduces to $m^{6}+m^{2}=0$, which has the solutions $m=0$ or $m=$ $(-1 \pm i) / \sqrt{2}$. The roots of $m$ that have a positive real part have been omitted. The $n=0$ contribution to the Fourier representation of the buoyancy becomes

$$
\begin{aligned}
\left.B(\forall Y, Z)\right|_{n=0}= & D_{0} \exp [(-1+i) Z / \sqrt{2}]+E_{0} \exp [(-1-i) Z / \sqrt{2}] \\
& +c_{1}+c_{2} \cdot Z,
\end{aligned}
$$

The last two terms are associated with the two identical roots $m=0$. They produce, respectively, a constant buoyancy and a buoyancy that increases with height. Both terms violate the boundary condition requiring that the buoyancy vanishes far above the surface, hence both constants $c_{1}$ and $c_{2}$ must be zero.

In order to evaluate the expression for the downslope velocity (A.12) we must revisit the steps leading to (A.9)-(A.11). From the latter equation we find that $\cos (0) g^{2} \epsilon_{0} e^{g Z}=0$, i.e. $g=0$. The particular solution $U_{p}$, however, is nonzero. Seeking a solution on the form (A.9) and using the new roots $m$, we obtain

$$
\nabla^{2} U_{p}=-i \delta_{2,0} D_{0} \exp [(-1+i) Z / \sqrt{2}]+i \delta_{3,0} E_{0} \exp [(-1-i) Z / \sqrt{2}] .
$$

Equating exponents with those in Eq. C.1 yields $\delta_{2,0}=i$ and $\delta_{3,0}=-i$. The $n=0$ contribution to the Fourier representation of $U$ is thus

$$
\left.\left.U\right|_{n=0}=i D_{0} \exp [(-1+i) Z / \sqrt{(} 2)\right]-i E_{0} \exp [(-1-i) Z / \sqrt{(2)}] .
$$


From the discussion on the surface boundary conditions (Appendix B) we know that the buoyancy at the surface is equal to $\mathcal{B}_{0}$, i.e. $\mathcal{B}_{0}=D_{0}+E_{0}$. The no-slip condition implies $0=i D_{0}-i E_{0}$. With these boundary conditions, the solution to (C.1) and (C.2) becomes

$$
\begin{aligned}
& \left.B(Y, Z)\right|_{n=0}=\mathcal{B}_{0} e^{-Z / \sqrt{2}} \cos (Z / \sqrt{2}), \quad \text { and } \\
& \left.U(Y, Z)\right|_{n=0}=-\mathcal{B}_{0} e^{-Z / \sqrt{2}} \sin (Z / \sqrt{2}) .
\end{aligned}
$$

The result should be compared to the Prandtl solution, which in terms of the non-dimensional variables introduced in Sect. 2, is

$$
\begin{aligned}
& B_{P r}(Z)=-e^{-Z / \sqrt{2}} \cos (Z / \sqrt{2}), \quad \text { and } \\
& U_{P r}(Z)=e^{-Z / \sqrt{2}} \sin (Z / \sqrt{2}) .
\end{aligned}
$$

The sign is changed in order to ensure consistency with the sign of $b_{0}$. In the Prandtl model the negatively buoyant surface is infinitely wide, i.e. the isolation parameter $\mathcal{R}$ is zero. Using $\mathcal{R}=0$ in Eq. B. 2 yields $\mathcal{B}_{0}=-1$ and $\mathcal{B}_{n}=0$ for $n>0$. This shows that the model solution reverts to the Prandtl solution for $\mathcal{R}=0$.

\section{References}

1. Oerlemans J (1998) The atmospheric boundary layer over melting glaciers. In: Clear and cloudy boundary layers. Netherlands Academy of Siences (KNAW), pp 129-153. ISBN 90-6984-235-1

2. Whiteman CD (2000) Mountain meteorology: fundamentals and applications. Oxford University Press. ISBN 0-19-513271-8

3. Haiden T, Whiteman CD (2005) Katabatic flow mechanisms on a low-angle slope. J Appl Meteorol 44:113-126

4. Nappo CJ, Rao K (1987) A model study of pure katabatic flows. Tellus 39A:61-71

5. Papadopoulos KH, Helmis CG, Soilemes AT, Kalogiros J, Papageorgas PG, Asimakopoulos DN (1997) The structure of katabatic flows down a simple slope. Q J R Meteorol Soc 123:1581-1601

6. Prandtl L (1942) Führer durch die Strömungslehre. Vieweg u. Sohn, Braunschweig

7. Grisogono B, Oerlemans J (2001) Katabatic flow: analytic solution for gradually varying eddy diffusivities. J Atmos Sci 58:3349-3354

8. Grisogono B, Oerlemans J (2001) A theory for the estimation of surface fluxes in simple katabatic flows. Q J R Meteorol Soc 127:2725-2739

9. Grisogono B (2003) Post-onset behaviour of the pure katabatic flow. Boundary-Layer Meteorol 107:157175

10. Gutman LN, Malbakhov VM (1964) On the theory of katabatic winds of Antarctica. Met Issled 9:150-155 (In Russian)

11. Lykosov VN, Gutman LN (1972) Turbulent boundary layer above a sloping underlying surface. Izv Acad Sci USSR, Atmos Ocean Phys 8:799-809

12. Gutman LN, Melgarejo JW (1981) On the laws of geostrophic drag and heat transfer over a slightly inclined terrain. J Atmos Sci 38:1714-1724

13. Egger J (1985) Slope winds and the axisymmetric circulation over Antarctica. J Atmos Sci 42:1859-1867

14. Stiperski I, Kavcic I, Grisogono B, Durran DR (2007) Including Coriolis effects in the Prandtl model for katabatic flow. Q J R Meteorol Soc 133:101-106

15. Kavcic I, Grisogono B (2007) Katabatic flow with Coriolis effect and gradually varying eddy diffusivity. Boundary-Layer Meteorol 125:377-387

16. Shapiro A, Fedorovich E (2008) Coriolis effects in homogeneous and inhomogeneous katabatic flows. Q J R Meteorol Soc 134:353-370

17. Egger J (1990) Thermally forced flows: theory. In: Blumen W (ed) Atmospheric processes over complex terrain. Am Met Soc

18. Egger J (1981) On the linear two-dimensional theory of thermally induced slope winds. Beitr Z Phys Atmos 54:465-481

19. Kondo H (1984) The difference of the slope wind between day and night. J Meteorol Soc Jpn 62:224-233 
20. Shapiro A, Fedorovich E (2007) Katabatic flow along a differentially cooled sloping surface. J Fluid Mech 571:149-175

21. Zilitinkevich SS, Elperin T, Kleeorin N, Rogachevskii I, Esau IN, Mauritsen T, Miles MW (2008) Turbulence energetics in stably stratified geophysical flows: Strong and weak mixing regimes. Q J R Meteorol Soc 134:793-799

22. Shapiro A, Fedorovich E (2004) Prandtl number dependence of unsteady natural convection along a vertical plate in a stably stratified fluid. Int J Heat Mass Transfer 47:4911-4927

23. Pope SB (2000) Turbulent flows. Cambridge University Press, Cambridge

24. Nieuwstadt FTM (1990) Direct and large-eddy simulation of free convection. In: Proc. 9th international heat transfer conference. Amer. Soc. Mech. Eng., New York

25. Fedorovich E, Nieuwstadt FTM, Kaiser R (2001) Numerical and laboratory study of a horizontally evolving convective boundary layer. Part 1 . Transition regimes and development of the mixed layer. J Atmos Sci 58:70-86

26. Shapiro A, Fedorovich E (2006) Natural convection in a stably stratified fluid along vertical plates and cylinders with temporally periodic surface temperature variations. J Fluid Mech 546:295-311

27. Axelsen SL, Van Dop H (2009) Large-eddy simulation of katabatic winds. Part I. Comparison with observations. Acta Geophys 57(4):803-836. doi:10.2478/s11600-009-0041-6

28. Van den Broeke MR (1997) Structure and diurnal variations of the atmospheric boundary layer over a mid-latitude glacier in the summer. Boundary-Layer Meteorol 83:183-205

29. Abramowitz M, Stegun IA (1964) Handbook of mathematical functions with formulas, graphs and mathematical tables. National Bureau of Standards Applied Mathematics Series No. 55. US Dept. of Commerce, Washington DC

30. Kreyszig E (1999) Advanced engeneering mathematics, 8th edn. Wiley and Sons, Inc. ISBN 0-471-33328-X 